\title{
Up-regulation expression and prognostic significance of Syntaxin4 in kidney renal clear cell carcinoma
}

\author{
Lishan $\mathrm{He}^{1^{*+}}$, Huiming Jiang ${ }^{2 \dagger}$, Zhenqiang Lai $^{3}$, Zhixiong Zhong ${ }^{4^{*}}$ and Zhanqin Huang ${ }^{5^{*}}$
}

\begin{abstract}
Background: Syntaxin4 (STX4) gene encodes the protein STX4, a member of soluble N-ethylmaleimide-sensitive factor attachment protein receptors protein, playing a vital role in cell invadopodium formation and invasion, which is associated with the malignant progression of various human cancers. However, the expression and prognostic significance of STX4 in kidney renal clear cell carcinoma (KIRC) remain to be investigated.

Methods: In this study, we collected the mRNA expression of STX4 in 535 KIRC patients from The Cancer Genome Atlasthrough the University of California Santa Cruz Xena database platform. Then we explored the expression of STX4 in KIRC, and the relationship with clinicopathological characteristics and prognostic value. Gene Ontology and Kyoto Encyclopedia of Genes and Genomes function enrichment analyses were used to explore the potential mechanism of STX4 in KIRC. qRT-PCR analysis was performed toverify the above results with real world tissue specimens.
\end{abstract}

Results: The results indicated that STX4 was up-expressed in KIRC, and were associated with higher histological grade, advanced stage, and poorer prognosis. Moreover, elevated STX4 expression is an independent risk factor for KIRC. qRT-PCR analysis showed that STX4 was significantly elevated in 10 paired of KIRC samples compared to normal samples. Functional enrichment analysis indicated that endo/exocytosis, autophagy, mTOR signaling pathway, and NOD-like receptor signaling pathway were enriched.

Conclusions: In summary, STX4 is constantly up-expressed in KIRC tissues, associated with a poor prognosis. We suggest that it can be an effective biomarker for the prognosis of KIRC and may be a novel therapeutic target in KIRC.

Keywords: Syntaxin4, Kidney renal clear cell carcinoma, Endo/exocytosis, Cell invasion, Prognostic

\footnotetext{
*Correspondence: lisaho518@163.com; zhongzhixiong@mzrmyy.com; zqhuang@stu.edu.cn

${ }^{\dagger}$ Lishan He and Huiming Jiang contributed equally to this work.

'Department of Clinical Pharmacy, Meizhou People's Hospital (Huangtang Hospital), Huangtang Road 63\#, Meijiang District, Meizhou, People's Republic of China 514031

${ }^{4}$ Center for Cardiovascular Diseases, Meizhou People's Hospital (Huangtang

Hospital), Huangtang Road 63\#, Meijiang District, Meizhou, People's Republic of China 514031

${ }^{5}$ Department of Pharmacology, Shantou University Medical College, Xinling

Road 22\#, Shantou, People's Republic of China 515041

Full list of author information is available at the end of the article
}

(c) The Author(s). 2021 Open Access This article is licensed under a Creative Commons Attribution 4.0 International License, which permits use, sharing, adaptation, distribution and reproduction in any medium or format, as long as you give appropriate credit to the original author(s) and the source, provide a link to the Creative Commons licence, and indicate if changes were made. The images or other third party material in this article are included in the article's Creative Commons licence, unless indicated otherwise in a credit line to the material. If material is not included in the article's Creative Commons licence and your intended use is not permitted by statutory regulation or exceeds the permitted use, you will need to obtain permission directly from the copyright holder. To view a copy of this licence, visit http://creativecommons.org/licenses/by/4.0/. The Creative Commons Public Domain Dedication waiver (http://creativecommons.org/publicdomain/zero/1.0/) applies to the data made available in this article, unless otherwise stated in a credit line to the data. 


\section{Background}

Kidney renal clear cell carcinoma (KIRC) is the main histological subtype of the renal cell carcinoma (RCC), accounting for $80-90 \%$ of patients [1]. KIRC was one of the ten leading cancer types for the estimated new cancer cases and deaths in the United States, and it had caused about 14,770 new deaths according to cancer statistics data in 2019 [2]. More than half of KIRC patients are a symptomless and diagnosed incidentally on imaging [3]. Although nephrectomy or targeted therapies had been implementation, approximately $30 \%$ of patients with localized tumor eventually develop metastases and the 5-year survival rate for patients with metastatic is less than $10 \%[4,5]$. The TNM stage is usually used as risk predictors for KIRC [6], but the outcomes for KIRC were heterogeneous in various aspects including clinicopathological, molecular, and cellular heterogeneity. Therefore, it is urgent to study the carcinogenesis and progression of KIRC and explore new useful molecular markers for prognosis.

Metastasis is a complex multicellular process that dependents on tumor cell invasion through the extracellular matrix, a supportive scaffold that acts to compartmentalize tissues [7]. Membrane trafficking of cellular cargo mediated in part by soluble $\mathrm{N}$ ethylmaleimide-sensitive factor attachment protein receptors (SNAREs), a family of membrane proteins that form complexes bridging apposed membranes and allowing membrane fusion [8]. Syntaxin4 (STX4) is one of SNAREs proteins implicated in the trafficking of membrane type-1 matrix metalloproteinase (MT1MMP) to the plasma membrane [9]. Recent research has shown that STX4 mediates invadopodium formation and tumor cell invasion [10]. However, it is unclear whether STX4 involved in the metastasis of KIRC. In this study, we explored and identified the STX4 associated with survival in patients with KIRC. We found that the SNAREs protein STX4 was positively correlated with malignant clinicopathological characteristics and was significantly related to overall survival (OS) in patients with KIRC. Most importantly, elevated STX4 expression is an independent risk factor for KIRC.

\section{Methods}

\section{Data extraction and identification of prognostic STX4 in KIRC}

The study is approved by Medical Ethics Committee of Meizhou People's Hospital (2020-CY-06) and in accordance with the ethical standards of the institutional and/ or national research committee and with the 1964 Helsinki declaration and its later amendments or comparable ethical standards. We collected the mRNA expression of STX4 and clinical data of 535 KIRC patients from The Cancer Genome Atlas (TCGA) through the
University of California Santa Cruz Xena database platform (https://xena.ucsc.edu/). Among them, 531 KIRC patients had information regarding survival. We matched the patients' clinical information and STX4 mRNA expression data. The different expression of STX4 was compared between 535 KIRC samples and 72 normal kidney samples. The correlation between the expression level of STX4 and clinicopathological characteristics in KIRC was also assessed. Kaplan-Meier (KM) survival curves was used for prognosis analysis using the $\mathrm{R}$ packages "survival" and "survminer", Log-rank test $P$ value $<0.05$ was chosen to be significantly different. Finally, we validated the results using the pan-cancer data from TCGA (32 other types of cancer) and qRT-PCR results of 10 paired of KIRC and normal real-world samples as internal and external validations, respectively.

\section{Quantitative reverse transcription polymerase chain reaction (qRT-PCR)}

To further validate the RNA-sequencing data obtained from TCGA, qRT-PCR analysis was performed to validate the expression of STX4 in 10 paired of KIRC and normal samples. We collected KIRC samples and paired adjacent normal samples from 10 patients who underwent nephrectomies or partial nephrectomies at Meizhou People's Hospital between 2019 and 2020. Informed consent was obtained from all patients. We extracted total RNA using the TRIzol ${ }^{\mathrm{TM}}$ reagent (Waltham, Massachusetts, USA). First-strand complementary DNA was synthesized equal amounts of total RNA $(4 \mu \mathrm{g})$ using the PrimeScript RT reagent kit (Takara Bio, Inc., Dalian, China) according to the manufacturer's instructions. Analyzed by The SYBR Green PCR kit (Takara Bio, Inc., Dalian, China) incorporation in PCR reactions involving specific primers and performed in the ABI 7500 fluorescent quantitative PCR system (Applied Biosystems Inc., Foster City, CA, USA). Glyceraldehyde 3-phosphate dehydrogenase (GAPDH) was used as the internal control. Thespecific primer sequences were as follows: STX4 (forward: CTGTCCCAGCAATTCGTGGAG; reverse: CCCAGCATTGGTGATCTTCAG), and GAPDH (forward: ATGACATCAAGAAGGTGGTG; reverse: CATACCAGGAAATGA GCTTG). The expression level was also calculated using the $2^{-\Delta \Delta C t}$ method.

\section{Functional and pathway enrichment analyses}

To identify biological functions in STX4 gene set, we carried out a Gene Ontology (GO) classification, which included the following categories: biological process, cellular component, and molecular functions. We firstly explored the co-expressed genes with the STX4 (correlation coefficient $\mathrm{r}>0.4, P<0.001$ ). Then we used the entire co-expressed genes matrix after preprocessing as a background, and performed GO 
functional enrichment analysis at online tools (http:// kobas.cbi.pku.edu.cn/) [11]. We explored the Kyoto Encyclopedia of Genes and Genomes (KEGG) analysis with the same method.

\section{Statistics}

All data processing and statistical analysis were performed using R (version 3.6.1; The R Foundation for Statistical Computing, Vienna, Austria), Strawberry Perl (version 5.30.1.1; http://strawberryperl.com/), and Statistical Pack/ age for Social Sciences (version 25.0; IBM, Armonk, New York, USA). Analysis of variance or $t$-test was used to compare the gene expression level among different subgroups. The differences were considered significant when $P<0.05$.

\section{Results}

STX4 expression was significantly up-regulated in KIRC

After matching the patients' clinical information and STX4 gene expression data, 535 patients with KIRC were enrolled in the study. The 535 patients' clinicopathological features are shown in Table 1. After analyzing the expression levels of STX4 in 535 KIRC samples and 72 normal kidney samples, we found that the expression of STX4 in KIRC tissues was obviously higher compared with normal tissues $(P<0.05)$ (Fig. 1A). To validate STX4 mRNA expression in KIRC tissues, we performed qRT-PCR in 10 paired tumor and normal samples. Compared with normal tissues, the expression of STX4 in KIRC tissues was significantly elevated by qRT-PCR result $(P<0.05)($ Fig. $1 \mathrm{~B}, \mathrm{C})$.

\section{Up-expression of STX4 predicts poor prognosis of KIRC}

Then we investigated the prognostic value of STX4 in KIRC. According to the median expression level of STX4, 531 patients with KIRC with survival information were divided into the high-STX4 and low-STX4 expression groups. As shown by the Kaplan-Meier (KM) survival analysis curve, there was a close relationship between the expression of STX4 and the survival of KIRC patients that the high expression of STX4 caused poor OS $(\mathrm{HR}=2.3, P<0.001$, Fig. $2 \mathrm{~A})$. We next investigated the relationship between STX4 expression and the clinicopathological characteristics of KIRC. Analysis results showed that the STX4 expression level was significantly related to several clinicopathological features of KIRC, including cancer status $(P=0.003)$, histological grade $(P<0.001)$, tumor size (T stage, $P<0.001)$, distant metastasis (M stage, $P<0.001$ ), and American Joint Committee on Cancer (AJCC) stage $(P<0.001)$ (Table 1). As shown in Fig. 2B, STX4 expression in KIRC tissues was significantly correlated with pathological grade $(P<0.001)$. Figure $2 \mathrm{C}$ showed the relationship between STX4 expression and different clinical stages, and the results suggested that STX4 expression was positively correlated with advanced clinical stage $(P<0.001)$.
Table 1 Correlations between the expression of STX 4 and clinicopathologic characteristics in KIRC

\begin{tabular}{|c|c|c|c|c|}
\hline \multirow[t]{2}{*}{ Characteristic } & \multirow[t]{2}{*}{ n (\%) } & \multicolumn{2}{|c|}{ Expression of STX4 (\%) } & \multirow{2}{*}{$\begin{array}{l}P \text { - } \\
\text { value }\end{array}$} \\
\hline & & High & Low & \\
\hline Total & $535(100)$ & $267(49.91)$ & $268(50.09)$ & \\
\hline Age & & & & 0.437 \\
\hline$Z A \leq 60$ years & $267(49.91)$ & $138(51.7)$ & $129(48.1)$ & \\
\hline$>60$ years & $268(50.09)$ & $129(48.3)$ & $139(51.9)$ & \\
\hline Gender & & & & 0.277 \\
\hline Female & $186(34.77)$ & $99(37.1)$ & $87(32.5)$ & \\
\hline Male & $349(65.23)$ & $168(62.9)$ & $181(67.5)$ & \\
\hline Cancer status & & & & 0.003 \\
\hline Tumor free & $336(62.8)$ & $155(58.1)$ & $181(67.5)$ & \\
\hline With tumor & $148(27.66)$ & $90(33.7)$ & $58(21.6)$ & \\
\hline Unknow & $51(9.53)$ & $22(8.2)$ & $29(10.8)$ & \\
\hline Race & & & & 0.191 \\
\hline White & $463(86.54)$ & $226(84.6)$ & $237(88.4)$ & \\
\hline Asian & $8(1.5)$ & $4(1.5)$ & $4(1.5)$ & \\
\hline Black & $57(10.65)$ & $35(13.1)$ & $22(8.2)$ & \\
\hline Unknow & $7(1.31)$ & $2(0.7)$ & $5(1.9)$ & \\
\hline Grade & & & & $<0.001$ \\
\hline G1 & $14(2.62)$ & $8(3.0)$ & $6(2.2)$ & \\
\hline G2 & $231(43.18)$ & $91(34.1)$ & $140(52.2)$ & \\
\hline G3 & 207 (38.69) & $119(44.6)$ & $88(32.8)$ & \\
\hline G4 & $75(14.02)$ & $48(18.0)$ & $27(10.1)$ & \\
\hline Unknow & $8(1.5)$ & $1(0.4)$ & $7(2.6)$ & \\
\hline T stage & & & & $<0.001$ \\
\hline $\mathrm{T} 1$ & $275(51.4)$ & $164(61.4)$ & $111(41.4)$ & \\
\hline $\mathrm{T} 2$ & 70 (13.08) & $32(12.0)$ & $38(14.2)$ & \\
\hline T3 & 179 (33.46) & $70(26.2)$ & $109(40.7)$ & \\
\hline T4 & $11(2.06)$ & $1(0.4)$ & $10(3.7)$ & \\
\hline N stage & & & & 0.616 \\
\hline No & $240(44.86)$ & $118(44.2)$ & $122(45.5)$ & \\
\hline N1 & 16 (2.99) & $9(3.4)$ & $7(2.6)$ & \\
\hline Unknow & $279(52.15)$ & $140(52.4)$ & 139 (51.9) & \\
\hline M stage & & & & $<0.001$ \\
\hline MO & $424(79.25)$ & $188(70.4)$ & $236(88.1)$ & \\
\hline M1 & 78 (14.58) & $52(19.5)$ & $26(9.7)$ & \\
\hline Unknow & $33(6.17)$ & $27(10.1)$ & $6(2.2)$ & \\
\hline AJCC stage & & & & $<0.001$ \\
\hline Stage I & $269(50.28)$ & $110(41.2)$ & $159(59.3)$ & \\
\hline Stage II & $58(10.84)$ & 29 (10.9) & 29 (10.8) & \\
\hline Stage III & $123(22.99)$ & $72(27.0)$ & $51(19.0)$ & \\
\hline Stage IV & $82(15.33)$ & $54(20.2)$ & $28(10.4)$ & \\
\hline Unknow & $3(0.56)$ & $2(0.7)$ & $1(0.4)$ & \\
\hline
\end{tabular}

KIRC Kidney renal clear cell carcinoma, AJCC American Joint Committee on Cancer 


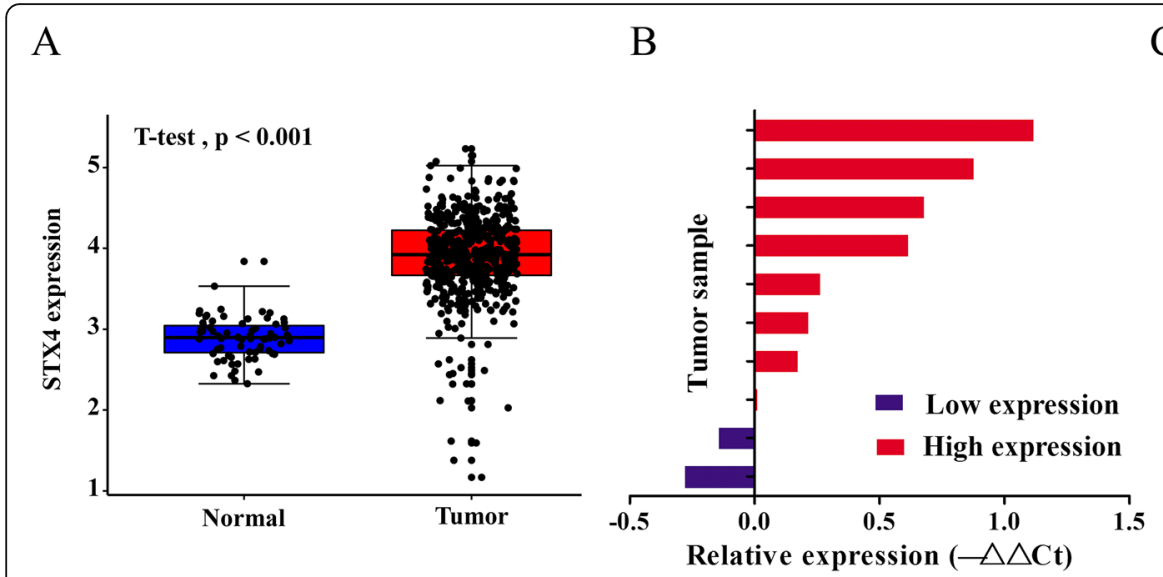

$\mathrm{C}$

Fig. 1 Expression of STX4 mRNA in human KIRC. (A) STX4 transcription level was significantly higher in 535 KIRC tissue samples than in 72 normal tissue samples. (B) STX4 was up-regulated in 8 of 10 KIRC tissue samples. (C) Compared with normal tissue samples, STX4 was significantly upregulated in tumor samples of 10 paired samples

To further validate the results, we explored the expression of STX4 in pan-cancer data (32 other cancer types) from TCGA as an internal validation. The "high" and "low" subgroups were always defined based on the mean expression value of STX4 in pan-cancer. According to the result of $\mathrm{KM}$ survival analysis, it is interesting that STX4 also played a prognostic role in cervical squamous cell carcinoma and endocervical adenocarcinoma, skin cutaneous melanoma, and uveal melanoma (Table 2). These results suggest that STX4 functions as a tumor promoter in KIRC.

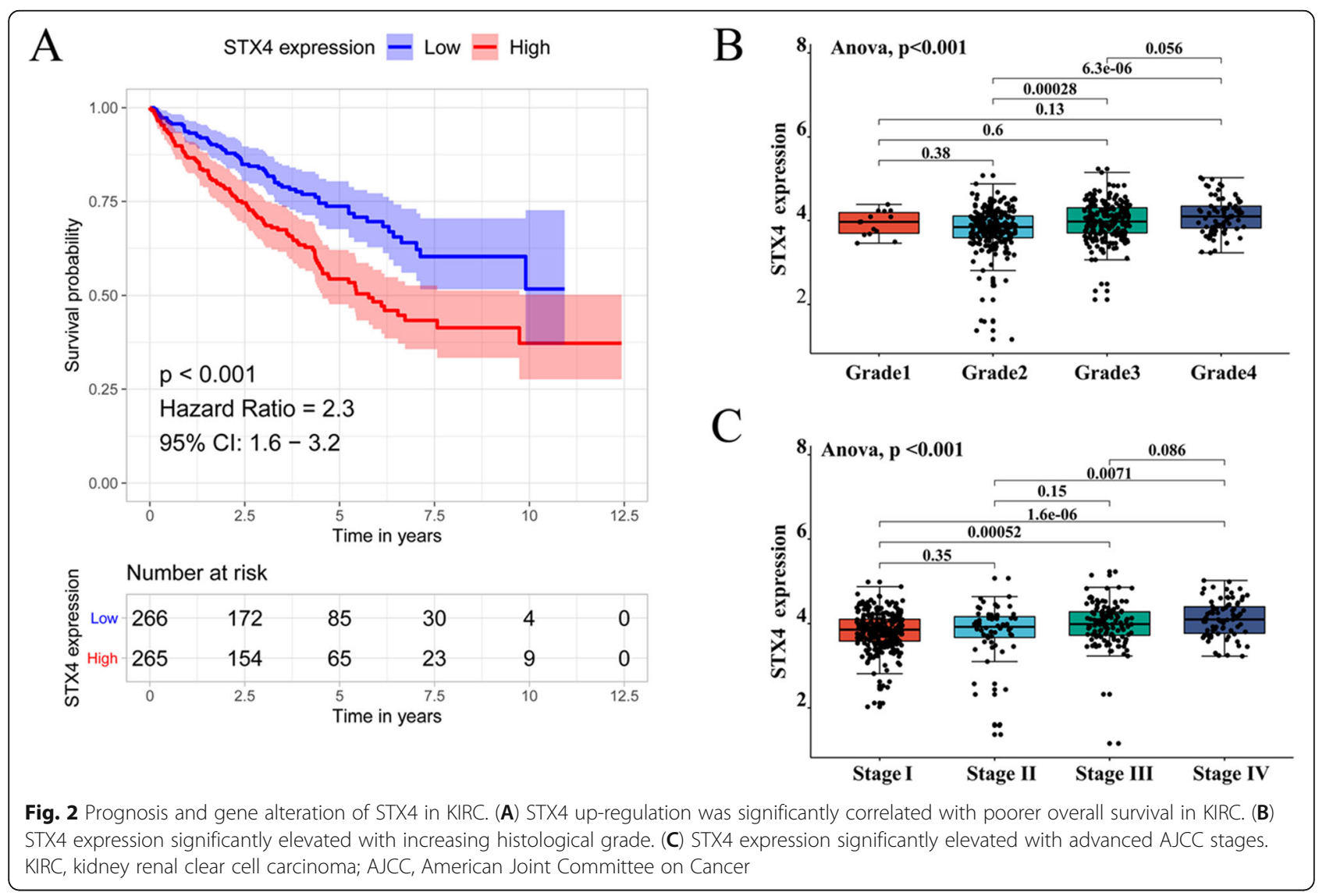


Table 2 Kaplan-Meier survival analysis for STX4 in pan-cancer (33 types of cancer from TCGA)

\begin{tabular}{|c|c|c|}
\hline Abbreviation & Detail & Log-rank test $p$ value \\
\hline ACC & Adrenocortical carcinoma & 0.277 \\
\hline BLCA & Bladder Urothelial Carcinoma & 0.702 \\
\hline BRCA & Breast invasive carcinoma & 0.954 \\
\hline CESC & Cervical squamous cell carcinoma and endocervical adenocarcinoma & 0.009 \\
\hline $\mathrm{CHOL}$ & Cholangio carcinoma & 0.675 \\
\hline COAD & Colon adenocarcinoma & 0.618 \\
\hline DLBC & Lymphoid Neoplasm Diffuse Large B-cell Lymphoma & 0.629 \\
\hline ESCA & Esophageal carcinoma & 0.309 \\
\hline GBM & Glioblastoma multiforme & 0.098 \\
\hline HNSC & Head and Neck squamous cell carcinoma & 0.438 \\
\hline $\mathrm{KICH}$ & Kidney Chromophobe & 0.133 \\
\hline KIRC & Kidney renal clear cell carcinoma & 0.000 \\
\hline KIRP & Kidney renal papillary cell carcinoma & 0.573 \\
\hline LAML & Acute Myeloid Leukemia & 0.185 \\
\hline LGG & Brain Lower Grade Glioma & 0.888 \\
\hline $\mathrm{LIHC}$ & Liver hepatocellular carcinoma & 0.102 \\
\hline LUAD & Lung adenocarcinoma & 0.801 \\
\hline LUSC & Lung squamous cell carcinoma & 0.936 \\
\hline MESO & Mesothelioma & 0.319 \\
\hline OV & Ovarian serous cystadenocarcinoma & 0.943 \\
\hline PAAD & Pancreatic adenocarcinoma & 0.733 \\
\hline PCPG & Pheochromocytoma and Paraganglioma & 0.453 \\
\hline PRAD & Prostate adenocarcinoma & 0.161 \\
\hline READ & Rectum adenocarcinoma & 0.766 \\
\hline SARC & Sarcoma & 0.442 \\
\hline SKCM & Skin Cutaneous Melanoma & 0.026 \\
\hline STAD & Stomach adenocarcinoma & 0.155 \\
\hline TGCT & Testicular Germ Cell Tumors & 0.315 \\
\hline THCA & Thyroid carcinoma & 0.445 \\
\hline THYM & Thymoma & 0.656 \\
\hline UCEC & Uterine Corpus Endometrial Carcinoma & 0.832 \\
\hline UCS & Uterine Carcinosarcoma & 0.248 \\
\hline UVM & Uveal Melanoma & 0.003 \\
\hline
\end{tabular}

\section{Independent prognostic analysis of STX4 in KIRC}

On the basis of KM survival analysis curve showing that KIRC patients with up-expression of STX4 had obviously poor OS, univariate and multivariate Cox regression analyses were used to further explored whether STX4 had an independent prognostic value in KIRC. The results showed that there was a significantly prognostic difference between the high-STX4 and low-STX4 expression groups in both univariate (HR, 1.743; 95\%CI, 1.279-2.374; $P<0.001)$ and multivariate (HR, 1.625; 95\%CI, 1.187-2.223; $P=0.002)$ Cox regression analyses, which suggested that STX4 was an independent prognostic factor in KIRC (Fig. 3A, B).

\section{Functional and pathway enrichment analyses}

GO functional enrichment analysis and KEGG analysis for STX4 were performed to investigate the molecular function and biological process of STX4. GO functional enrichment analysis for proteins interacting with STX4 demonstrated that the molecular functions cover protein binding processes, protein transport processes, and endosomal transport processes, autophagosome, and ubiquitin binding (Table 3). KEGG analysis revealed that the endocytosis, lysosome, and proteasome were enriched (Table 4). Meanwhile, mTOR signaling pathway, NOD-like receptor signaling pathway, and SNARE interactions in vesicular transport were also enriched (Table 4). 


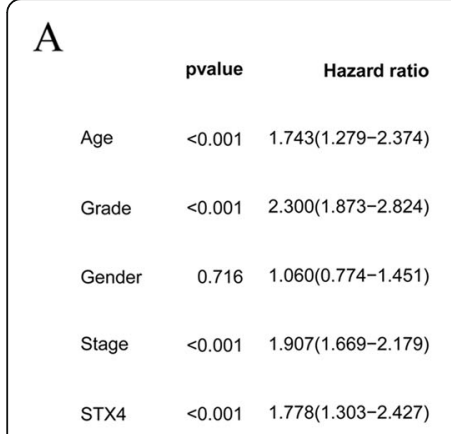

Fig. 3 Forest plot of Cox regression analysis in KIRC. (A) Forest plot of univariate Cox regression analysis. (B) Forest plot of multivariate Cox regression analysis

\section{Discussion}

KIRC was one of the highest incidence subtypes of the RCC with poor prognosis. Due to limited predictors assessing the risk because of tumor heterogeneity, a part of KIRC patients with poor prognosis might be miss aggressive treatment due to a delay in diagnosis, which would decrease the survival rate of patients with KIRC to some extent. Hence, it is crucial to identify new specific prognosis predictors for KIRC.

STX4 is one of the SNAREs proteins and is involved in cell invadopodium formation and tumor cell invasion $[9,10]$. Research has shown that STX4 also plays an

Table $3 \mathrm{GO}$ function analysis for proteins interacting with STX4

\begin{tabular}{|c|c|c|}
\hline Pathway description & Pathway ID & $P$-Value \\
\hline protein binding & GO:0005515 & 8.33E-89 \\
\hline cytosol & GO:0005829 & $2.27 \mathrm{E}-32$ \\
\hline cytoplasm & GO:0005737 & $4.31 \mathrm{E}-30$ \\
\hline identical protein binding & GO:0042802 & 4.51E-10 \\
\hline spliceosomal complex & GO:0005681 & $1.20 \mathrm{E}-08$ \\
\hline molecular_function & GO:0003674 & $5.40 \mathrm{E}-06$ \\
\hline plasma membrane & GO:0005886 & 8.27E-06 \\
\hline membrane & GO:0016020 & $8.51 \mathrm{E}-06$ \\
\hline protein transport & GO:0015031 & $9.29 \mathrm{E}-06$ \\
\hline protein kinase binding & GO:0019901 & $1.06 \mathrm{E}-05$ \\
\hline intracellular signal transduction & GO:0035556 & $1.50 \mathrm{E}-05$ \\
\hline protein phosphorylation & GO:0006468 & $5.34 \mathrm{E}-05$ \\
\hline tumor necrosis factor-mediated signaling pathway & GO:0033209 & $7.14 \mathrm{E}-05$ \\
\hline intracellular membrane-bounded organelle & GO:0043231 & 0.000155436 \\
\hline transcription export complex & GO:0000346 & 0.000206682 \\
\hline apoptotic process & GO:0006915 & 0.000229098 \\
\hline endosomal transport & GO:0016197 & 0.000322781 \\
\hline intracellular protein transport & GO:0006886 & 0.000350599 \\
\hline U2-type prespliceosome & GO:0071004 & 0.000478136 \\
\hline autophagosome & GO:0005776 & 0.000499235 \\
\hline microtubule organizing center & GO:0005815 & 0.000522493 \\
\hline endomembrane system & GO:0012505 & 0.000786406 \\
\hline signal transduction & GO:0007165 & 0.000824551 \\
\hline ubiquitin binding & GO:0043130 & 0.000930267 \\
\hline
\end{tabular}


Table 4 KEGG function analysis for proteins interacting with STX4

\begin{tabular}{lll}
\hline Pathway description & description ID & P-Value \\
\hline Spliceosome & hsa03040 & $1.00 \mathrm{E}-06$ \\
Endocytosis & hsa04144 & 0.000147263 \\
Choline metabolism in cancer & hsa05231 & 0.000232401 \\
mRNA surveillance pathway & hsa03015 & 0.000322781 \\
Homologous recombination & hsa03440 & 0.000405621 \\
Glycerophospholipid metabolism & hsa00564 & 0.000573028 \\
Lysosome & hsa04142 & 0.000672647 \\
RNA transport & hsa03013 & 0.000864392 \\
Necroptosis & hsa04217 & 0.001676903 \\
NOD-like receptor signaling pathway & hsa04621 & 0.001929387 \\
C-type lectin receptor signaling pathway & hsa04625 & 0.002830637 \\
Epstein-Barr virus infection & hsa05169 & 0.00314403 \\
GnRH signaling pathway & hsa04912 & 0.003202064 \\
Phospholipase D signaling pathway & hsa04072 & 0.003763014 \\
VEGF signaling pathway & hsa04370 & 0.003815766 \\
mTOR signaling pathway & hsa04150 & 0.005035986 \\
Non-small cell lung cancer & hsa05223 & 0.007316192 \\
Base excision repair & hsa03410 & 0.008263833 \\
Pathways in cancer & hsa05200 & 0.008968622 \\
Proteasome & hsa03050 & 0.009338017 \\
SNARE interactions in vesicular transport & hsa04130 & 0.009352469 \\
\hline
\end{tabular}

important role in several cancers. A research had identified the exocytosis mediator proteins STX4 in the peripheral blood neutrophils of patients with chronic myeloid leukemia early in 2004 [12]. Breast cancer showed the strongest correlation with the expression of STX4, the latter was associated with reduced patient survival in high expression [13]. Extracellular STX4 triggers the differentiation program in teratocarcinoma F9 cells that impacts cell adhesion properties [14]. It still had not study reveal the effect of STX4 in KIRC. Here, we screened out available datasets associated with KIRC from public databases to confirm the function of STX4 on the oncoming, progression, and prognosis of KIRC. In this study, STX4 upregulation was significantly associated with unfavorable clinicopathological features in KIRC, such as higher histological grade, larger tumor size, distant metastasis, and advanced AJCC stage. KM survival analysis curve showed that STX4 expression had maintained a high level with a poor OS. Furthermore, univariate and multivariate Cox regression analyses confirmed that STX4 played an independent prognostic role in KIRC. The qRT-PCR results from 10 paired of KIRC and normal real-world samples further confirmed the up-regulation of STX4 in KIRC. This suggested that STX4 could be identified as a potential prognostic biomarker in KIRC.
We used data of 32 other types of cancer from TCGA to validate the aforementioned results. As the pancancer analysis result shown that survival differences of STX4 existed in several types of cancer. All results were consistent and suggested that STX4 may serve as a tumor promoter in KIRC.

Further research into how STX4 influences patients' survival via GO functional enrichment analysis and KEGG analysis. These analyses demonstrated that the molecular functions of STX4 cover protein binding processes, protein transport processes, endosomal transport processes, and endocytosis, which suggest that STX4 influences the endo/exocytosis of the tumor. The fusion of secretory vesicles and subsequent protein exocytosis are the important mechanism of cancer cells metastasis [7]. Alterations of endo/exocytic proteins have long been associated with malignant transformation, and genes encoding membrane trafficking proteins have been identified as bona fide drivers of tumorigenesis [15]. Results had demonstrated that STX4 defines a domain for activity-dependent exocytosis in dendritic spines $[16,17]$ STX4 mediated trafficking of MT1-MMP during invadopodium formation and tumor cell invasion [10, 18]. Autophagosome, lysosome, mTOR signaling pathway, and NOD-like receptor signaling pathway were also enriched by GO and KEGG analyses. Autophagy is a lysosomal-dependent pathway for intracellular degradation, leading to the basal turnover of cell components and providing energy and macromolecular precursors. Autophagy has opposing, context-dependent roles in cancer, and interventions to both stimulate and inhibit autophagy have been proposed as cancer therapies [19]. In addition, mTOR signaling pathway and NOD-like receptor signaling pathway play a crucial role in regulating autophagy [20-22]. STX2 to block STX3- and STX4mediated fusion of zymogen granules with the plasma membrane and exocytosis and prevent binding of ATG16L1 to clathrin, which contributes to induction of autophagy [23]. Thus, we speculated that STX4 may promote the tumor progression and influence the prognosis of KIRC by regulating endo/exocytosis, and autophagy.

This study has several limitations, although it is the first to discover the potential prognostic value of STX4 in KIRC. First, although differential STX4 expression was detected between tumor and normal real-world samples, the prognostic implication of this finding has not been demonstrated. Second, only transcriptomics expression of STX4 with clinical data was analyzed to predict OS in this study. Third, the underlying mechanisms of STX4 in KIRC remain unclear, only function enrichment analyses were performed. Therefore, additional data and samples are necessary to confirm the results of this study. Future research is required to explore the detailed molecular mechanism of STX4 in KIRC. 
In conclusion, this study demonstrated that STX4 is a key survival-associated marker in KIRC. With a potential role in endo/exocytosis, STX4 may be a novel therapeutic target in patients with KIRC.

\section{Abbreviations}

KIRC: Kidney renal clear cell carcinoma; OS: Overall survival; TCGA: The Cancer Genome Atlas; PresSTIGE: Predicting Specific Tissue Interactions of Genes and Enhancers; qRT-PCR: Quantitative reverse transcription polymerase chain reaction; GAPDH: glyceraldehyde 3-phosphate dehydrogenase; GO: Gene Ontology; KEGG: Kyoto Encyclopedia of Genes and Genomes;

AJCC: American Joint Committee on Cancer

\section{Acknowledgements}

Not applicable.

\section{Authors' contributions}

Conceived and designed the experiments: ZQ H, ZX Z. Performed the experiments: LS H, ZQ L. Analyzed the data, generated the tables, and figures: HM J. Contributed reagents/materials/analysis tools and expertise: LS H. Wrote the manuscript: LS H. Revised the manuscript: LS H, HM J, ZQ H. All authors approved the submitted version.

\section{Funding}

This work was supported by the Peiyu Project of Meizhou People's Hospital (Grant/Award No. PY-C2019011) and (Grant/Award No. PY-C2020004).

\section{Availability of data and materials}

The datasets generated and/or analysed during the current study are available in the [The Cancer Genome Atlas (TCGA) through the University of California Santa Cruz Xena database platform] repository, (https://xena.ucsc. edu/).

\section{Declarations}

\section{Ethics approval and consent to participate}

The study is approved by Medical Ethics Committee of Meizhou People's Hospital (2020-CY-06) and in accordance with the ethical standards of the institutional and/or national research committee and with the 1964 Helsinki declaration and its later amendments or comparable ethical standards. Informed consent was obtained from all patients.

\section{Consent for publication}

Not applicable.

\section{Competing interests}

The authors declare that they have no competing interests.

\section{Author details}

'Department of Clinical Pharmacy, Meizhou People's Hospital (Huangtang Hospital), Huangtang Road 63\#, Meijiang District, Meizhou, People's Republic of China 514031. 'Department of Urology, Meizhou People's Hospital (Huangtang Hospital), Meizhou 514031, China. ${ }^{3}$ Pharmacy Intravenous Admixture Services, Meizhou People's Hospital (Huangtang Hospital), Meizhou 514031, China. ${ }^{4}$ Center for Cardiovascular Diseases, Meizhou People's Hospital (Huangtang Hospital), Huangtang Road 63\#, Meijiang District, Meizhou, People's Republic of China 514031. ${ }^{5}$ Department of Pharmacology, Shantou University Medical College, Xinling Road 22\#, Shantou, People's Republic of China 515041.

Received: 15 May 2021 Accepted: 23 August 2021

Published online: 06 September 2021

\section{References}

1. Ljungberg B, Bensalah K, Canfield S, Dabestani S, Hofmann F, Hora M, et al. EAU guidelines on renal cell carcinoma: 2014 update. Eur Urol. 2015;67(5): 913-24. https://doi.org/10.1016/j.eururo.2015.01.005.

2. Siegel RL, Miller KD, Jemal A. Cancer statistics, 2019. CA Cancer J Clin. 2019; 69(1):7-34. https://doi.org/10.3322/caac.21551.
3. Gray RE, Harris GT. Renal cell carcinoma: diagnosis and management. Am Fam Physician. 2019;99(3):179-84.

4. Hsieh JJ, Purdue MP, Signoretti S, Swanton C, Albiges L, Schmidinger M, et al. Renal cell carcinoma. Nat Rev Dis Primers. 2017;3(1):17009. https://doi. org/10.1038/nrdp.2017.9.

5. Mitsui Y, Shiina H, Kato T, Maekawa S, Hashimoto Y, Shiina M, et al. Versican promotes tumor progression, metastasis and predicts poor prognosis in renal carcinoma. Mol Cancer Res. 2017;15(7):884-95. https://doi.org/10.11 58/1541-7786.MCR-16-0444.

6. Motzer RJ, Jonasch E, Agarwal N, Beard C, Bhayani S, Bolger GB, et al. National comprehensive cancer network. Kidney cancer, version 3. 2015. J Natl Compr Cancer Netw. 2015;13(2):151-9. https://doi.org/10.6004/jnccn.2015.0022.

7. Stephens DC, Harris DA. Organizing 'Elements': facilitating exocytosis and promoting metastasis. Trends Cancer. 2020;6(4):273-6. https://doi.org/10.101 6/j.trecan.2020.01.020.

8. Chen YA, Scheller RH. SNARE-mediated membrane fusion. Nat Rev Mol Cell Biol. 2001;2(2):98-106. https://doi.org/10.1038/35052017.

9. Miyata T, Ohnishi H, Suzuki J, Yoshikumi Y, Ohno H, Mashima H, et al. Involvement of syntaxin 4 in the transport of membrane-type 1 matrix metalloproteinase to the plasma membrane in human gastric epithelial cells. Biochem Biophys Res Commun. 2004;323(1):118-24. https:/doi.org/10.1016/j.bbrc.2004.08.064.

10. Brasher MI, Martynowicz DM, Grafinger OR, Hucik A, Shanks-Skinner E, Uniacke J, et al. Interaction of Munc18c and syntaxin4 facilitates invadopodium formation and extracellular matrix invasion of tumor cells. J Biol Chem. 2017; 292(39):16199-210. https://doi.org/10.1074/jbc.M117.807438.

11. Xie C, Mao X, Huang J, Ding Y, Wu J, Dong S, et al. KOBAS 2.0: a web server for annotation and identification of enriched pathways and diseases. Nucleic Acids Res. 2011;39:W316-22.

12. Nuyanzina VA, Nabokina SM. Identification of exocytosis mediator proteins in peripheral blood neutrophils of patients with chronic myeloid leukemia. Bull Exp Biol Med. 2004;137(4):361-3. https://doi.org/10.1023/B:BEBM.0000035130.80245.13.

13. Althubiti M, Lezina L, Carrera S, Jukes-Jones R, Giblett SM, Antonov A, et al. Characterization of novel markers of senescence and their prognostic potential in cancer. Cell Death Dis. 2014;5(11):e1528. https://doi.org/10.1038/ cddis.2014.489.

14. Hagiwara N, Kadono N, Miyazaki T, Maekubo K, Hirai Y. Extracellular syntaxin4 triggers the differentiation program in teratocarcinoma F9 cells that impacts cell adhesion properties. Cell Tissue Res. 2013;354(2):581-91. https://doi.org/10.1007/s00441-013-1680-0.

15. Lanzetti L, Di Fiore PP. Behind the scenes: Endo/exocytosis in the Acquisition of Metastatic Traits. Cancer Res. 2017;77(8):1813-7. https://doi. org/10.1158/0008-5472.CAN-16-3403.

16. Kennedy MJ, Davison IG, Robinson CG, Ehlers MD. Syntaxin-4 defines a domain for activity-dependent exocytosis in dendritic spines. Cell. 2010; 141(3):524-35. https://doi.org/10.1016/j.cell.2010.02.042

17. Mohanasundaram P, Shanmugam MM. Role of syntaxin 4 in activitydependent exocytosis and synaptic plasticity in hippocampal neurons. Sci Signal. 2010;3:jc7.

18. Williams KC, MCNeilly RE, Coppolino MG. SNAP23, Syntaxin4, and vesicleassociated membrane protein 7 (VAMP7) mediate trafficking of membrane type 1-matrix metalloproteinase (MT1-MMP) during invadopodium formation and tumor cell invasion. Mol Biol Cell. 2014:25(13):2061-70. https://doi.org/10.1091/mbc.e13-10-0582.

19. Levy JMM, Towers CG, Thorburn A. Targeting autophagy in cancer. Nat Rev Cancer. 2017;17(9):528-42. https://doi.org/10.1038/nrc.2017.53.

20. Kim YC, Guan KL. mTOR: a pharmacologic target for autophagy regulation. $J$ Clin Invest. 2015;125:25-32.

21. Kim YK, Shin JS, Nahm MH. NOD-like receptors in infection, immunity, and diseases. Yonsei Med J. 2016;57(1):5-14. https://doi.org/10.3349/ymj.2016. 57.1.5.

22. Travassos LH, Carneiro LA, Ramjeet M, Hussey S, Kim YG, Magalhães JG, et al. Nod1 and Nod2 direct autophagy by recruiting ATG16L1 to the plasma membrane at the site of bacterial entry. Nat Immun. 2010;11(1):5562. https://doi.org/10.1038/ni.1823.

23. Dolai S, Liang T, Orabi Al, Holmyard D, Xie L, Greitzer-Antes D, et al. Pancreatitis-induced depletion of Syntaxin 2 promotes autophagy and increases Basolateral exocytosis. Gastroenterology. 2018;154:1805-1821.e5.

\section{Publisher's Note}

Springer Nature remains neutral with regard to jurisdictional claims in published maps and institutional affiliations. 